Memorias del VIII Encuentro Nacional de Experiencias en Enseñanza de la Biología y la Educación Ambiental. III Congreso Nacional de Investigación en Enseñanza de la Biología.

\title{
CONTRIBUCIONES DE LOS TRABAJOS PRÁCTICOS EN LA CONSTRUCCIÓN DEL CONCEPTO SISTEMA NERVIOSO
}

\section{CONTRIBUTIONS OF PRACTICAL WORK IN BUILDING CONCEPT NERVOUS SYSTEM}

Martínez Parra, Miguel Angel ${ }^{12 ;}$ Rangel Silva, María Natalia ${ }^{13 ;}$ Cepeda Benavides, Wilson ${ }^{14^{*}}$

\section{RESUMEN}

Sin duda, el trabajo práctico y, en particular, la actividad de laboratorio constituyen un hecho diferencial propio de la enseñanza de las ciencias. En el presente trabajo, se tomó como punto focal la implementación de trabajos prácticos con el fin de apoyar la construcción del concepto sistema nervioso a partir de dos sesiones de clase y una práctica de laboratorio. Se tuvo en cuenta como punto de partida las ideas previas que presentaban los estudiantes en cuanto a las estructuras del sistema nervioso humano y a partir de estas ideas de realizo una teorización acerca del sistema nervioso comparado donde se abordaron diferentes grupos taxonómicos, la cual dio soporte a la realización del ejerció práctico el cual consistió en la disección de un encéfalo de ave. Se evidencian aspectos favorables en la capacidad de establecer relaciones entre teoría y práctica, mediante la disección del encéfalo de un ave. El grueso de los estudiantes dio cuenta de las estructuras y funciones del sistema nervioso. Finalmente, se logró por medio de los trabajos prácticos contribuir a la construcción del concepto sistema nervioso de tal manera que los estudiantes lograban relacionar sus estructuras con el funcionamiento del cuerpo.

Palabras Clave: Ideas previas, sistema nervioso comparado, trabajos prácticos.

\section{ABSTRACT}

Undoubtedly, the practical work and, in particular laboratory activity constitute a proper differential fact of science education. In this paper, it was taken as a focal point to implement practical work in order to support the construction of the nervous system concept from two classroom sessions and lab. The previous ideas that the students had regarding the structures of the human nervous system and from these ideas made a theorizing about nervous system where different taxa compared addressed, which gave support was taken into account as a starting point the realization of practical served which consisted of dissecting a bird brain. Favorable aspects are

Estudiantes Licenciatura en Biología. Universidad Distrital Francisco José de Caldas

2. miamartinezp@correo.udistrital.edu.co

3. mnrangels@correo.udistrital.edu.co

4.wcepedab@correo.udistrital.edu.co 
Memorias del VIII Encuentro Nacional de Experiencias en Enseñanza de la Biología y la Educación Ambiental. III Congreso Nacional de Investigación en Enseñanza de la Biología.

evident in the ability to establish relationships between theory and practice, by dissecting the brain of a bird. The bulk of the students realized the structures and functions of the nervous system. Finally, it was achieved through practical work contribute to the construction of the concept nervous system so that students were able to relate their structure to the functioning of the body.

Keywords: Pre Ideas, compared nervous system , practical work

\section{INTRODUCCION}

La enseñanza de las ciencias no se debe limitar solamente a la explicación de contenidos a los estudiantes, sino que con esta se debe acercar al estudiante al trabajo científico, ya que este permitirá fortalecer las construcciones de los conceptos con los estudiantes, además de generar hábitos hacia las prácticas relacionadas con el campo científico (García; Mondelo y Martínez, 1995). Aunque estos trabajos no se deben restringir a la corroboración de la teoría (González, 1992), se debe tener en cuenta el planteamiento de hipótesis por parte de los estudiantes, una contextualización teórica, un análisis de los datos y una obtención de conclusiones por parte de los estudiantes, esto permitirá construcción conceptual la cual se enmarca dentro del quehacer científico y, por otra parte, demanda una formación docente específica (Furió y Gil 1989, Lledó y Cañal 1993, Olson 1990, Tamir 1989). Para lograr algún tipo de cambio metodológico del profesorado es indispensable partir de sus propias concepciones (Gil et al. 1991). Los trabajos prácticos juegan entonces un papel esencial en la construcción de conceptos. La clasificación de los trabajos prácticos ha sido preocupación de autores como Aureli Caamaño (2004), que propone una clasificación para estos (Ver tabla 1), según su finalidad, su campo de acción. Respecto a lo anterior, el principal objetivo de este trabajo es evidenciar cómo los trabajos prácticos contribuyen a la construcción del concepto sistema nervioso, a partir del reconocimiento del sistema nervioso de las aves, y comparándolo con el del Hombre.

Tabla 1. Clasificación de los trabajos prácticos según Caamaño (2004)

\begin{tabular}{|l|l|}
\hline \multicolumn{1}{|c|}{$\begin{array}{c}\text { TRABAJOS } \\
\text { PRACTICOS }\end{array}$} & \multicolumn{1}{|c|}{ DESCRIPCIÓN } \\
\hline Experiencias. & $\begin{array}{l}\text { Actividades prácticas destinadas a obtener una familiarización } \\
\text { perceptiva con los fenómenos. }\end{array}$ \\
\hline $\begin{array}{l}\text { Experimentos } \\
\text { ilustrativos. }\end{array}$ & $\begin{array}{l}\text { Están destinados a interpretar un fenómeno, ilustrar un principio o } \\
\text { mostrar una relación entre variables, Pueden constituir una } \\
\text { aproximación cualitativa o cuantitativa al fenómeno. }\end{array}$ \\
\hline $\begin{array}{l}\text { Ejercicios } \\
\text { prácticos. }\end{array}$ & $\begin{array}{l}\text { Son actividades diseñadas para aprender determinados } \\
\text { procedimientos o destrezas, o para realizar experimentos cuantitativos } \\
\text { que ilustren o corroboren la teoría. Estas pueden ser: }\end{array}$ \\
\hline Investigaciones. & $\begin{array}{l}\text { Son trabajos encaminados a resolver un problema teórico o práctico } \\
\text { mediante el diseño y la realización de un experimento y la evaluación } \\
\text { del resultado. }\end{array}$ \\
\hline
\end{tabular}


Memorias del VIII Encuentro Nacional de Experiencias en Enseñanza de la Biología y la Educación Ambiental. III Congreso Nacional de Investigación en Enseñanza de la Biología.

\section{METODOLOGÍA}

Se implementaron 3 sesiones de clases (Ver tabla 2.) sobre el sistema nervioso comparado, se tuvo en cuenta las ideas previas de los estudiantes. Como ejercicio de ideas previas, se realizó una guía que permitió, por un lado, la evaluación ${ }^{\dagger}$ de temas, y por otro el conocimiento de los estudiantes del sistema nervioso comparado. A partir de ello, se llevó a cabo una fase de teorización en la cual se relacionaron diversos grupos. Se realizó la disección de un encéfalo de ave; donde se debía establecer las semejanzas y diferencias entre el sistema nervioso del hombre y el de un ave.

Tabla 2. Sesiones de clase con sus objetivos y las actividades realizadas.

\begin{tabular}{|c|c|c|c|}
\hline $\begin{array}{l}\mathrm{N}^{\circ} \text { de } \\
\text { sesión }\end{array}$ & Tema & Objetivos & Actividad \\
\hline 1 & $\begin{array}{l}\text { Sistema } \\
\text { nervioso y } \\
\text { sentidos. }\end{array}$ & $\begin{array}{l}\text { Evaluar cualitativa } \quad \text { y } \\
\text { cuantitativamente } \\
\text { comprensión de los temas } \\
\text { sistema nervioso y sentidos por } \\
\text { parte de los estudiantes. }\end{array}$ & $\begin{array}{l}\text { Evaluación - Ejercicio ideas } \\
\text { previas (Ver Anexo 1) }\end{array}$ \\
\hline 2 & $\begin{array}{l}\text { Sistema } \\
\text { nervioso } \\
\text { comparado. }\end{array}$ & $\begin{array}{l}\text { Relacionar el sistema nervioso } \\
\text { de diferentes grupos } \\
\text { taxonómicos. }\end{array}$ & $\begin{array}{l}\text { Teorización mediante una } \\
\text { presentación con diapositivas y } \\
\text { una guía, con las principales } \\
\text { diferencias y semejanzas entre } \\
\text { el sistema nervioso de los } \\
\text { grupos taxonómicos (Ver } \\
\text { Anexo 2) }\end{array}$ \\
\hline 3 & $\begin{array}{l}\text { Sistema } \\
\text { nervioso } \\
\text { comparado } \\
\text { Aves - } \\
\text { Hombre }\end{array}$ & $\begin{array}{l}\text { Lograr acercamiento al trabajo } \\
\text { en laboratorio. } \\
\text { Realizar disección de un } \\
\text { encéfalo de ave. } \\
\text { Identificar semejanzas y } \\
\text { diferencias entre el sistema } \\
\text { nervioso de Aves y del } \\
\text { Hombre. }\end{array}$ & $\begin{array}{l}\text { Reconocimiento del material de } \\
\text { laboratorio a utilizar. Disección } \\
\text { del encéfalo de Ave. }\end{array}$ \\
\hline
\end{tabular}

Este trabajo se desarrolló en el Instituto Técnico Industrial Francisco José de Caldas (ITI), con un grupo de 38 estudiantes con edades oscilantes entre los 12 y 15 años pertenecientes al grado 812 de la Jornada Tarde. Sin embargo para esta investigación se tuvo en cuenta solo 6 estudiantes los cuales fueron escogidos según su participación de la siguiente manera: Estudiante 1 y 2 participación activa; Estudiante 3 y 4 participación media; estudiante 5 y 6 participación pasiva.

\footnotetext{
† La guía realizada se planteó como un ejercicio de evaluación, sin embargo fue relevante para identificar las ideas previas sobre sistema nervioso comparado que presentaban los estudiantes.
} 
Memorias del VIII Encuentro Nacional de Experiencias en Enseñanza de la Biología y la Educación Ambiental. III Congreso Nacional de Investigación en Enseñanza de la Biología.

\section{RESULTADOS Y DISCUSIÓN}

En esta discusión se analizaran las 3 sesiones de clases, con las preguntas propuestas y las respuestas que brindaron los estudiantes.

Sesión 1: En los estudiantes se pudo evidenciar las confusiones en cuanto a algunas funciones del sistema nervioso central. Ejemplo de ello es la pregunta uno del instrumento que fue implementado "Si una de las funciones del oído es encargarse del equilibrio ¿cómo es posible que un sordo o un sordo ciego puedan caminar con normalidad?" En la cual se observaba que en las respuestas de la mayoría de los estudiantes se confundía la función del oído interno, con la del oído medio (Ver tabla 3), además de no establecer relaciones con otros órganos. De los estudiantes 3 presentaron respuestas de tipo puntual (Giordan, 1987).

Una segunda pregunta "Colocar las partes del sistema nervioso central señaladas" permitió observar las confusiones estructurales que tenían en cuanto a las partes del encéfalo humano. Los estudiantes 1, 4 y 5 lograron establecer funciones del oído medio, mientras, los estudiantes 2, 3, 4, 5 y 6 mostraron falencias en cuanto a una definición concreta de la función relacionándola con otros órganos del cuerpo, la mayoría de ellos igualmente tuvieron errores en la ubicación de partes del sistema nervioso central. A partir de esto se ve la necesidad de realizar un trabajo práctico.

Tabla 3. Respuestas de los estudiantes que se tuvieron en cuenta en el primer instrumento

\begin{tabular}{|l|l|}
\hline \multicolumn{1}{|c|}{ SESIÓN 1 } \\
\hline ESTUDIANTE & \multicolumn{1}{|c|}{ RESPUESTA } \\
\hline Estudiante 1 & $\begin{array}{l}\text { "Porque el equilibrio está dado por el cerebelo, y el afectado en una } \\
\text { persona sorda es el oído medio" }\end{array}$ \\
\hline Estudiante 2 & "Porque todavía le sirve el oído externo" \\
\hline Estudiante 3 & "Porque los otros sentidos se agudizan" \\
\hline Estudiante 4 & $\begin{array}{l}\text { "La parte dañada en una persona sorda es diferente a la parte que controla } \\
\text { el equilibrio" }\end{array}$ \\
\hline Estudiante 5 & "Porque lo que se daña es el oído medio no el interno" \\
\hline Estudiante 6 & "Porque los oídos no tienen que ver con los músculos" \\
\hline \multicolumn{2}{|c|}{ Estructura** } \\
\hline
\end{tabular}


Memorias del VIII Encuentro Nacional de Experiencias en Enseñanza de la Biología y la Educación Ambiental. III Congreso Nacional de Investigación en Enseñanza de la Biología.

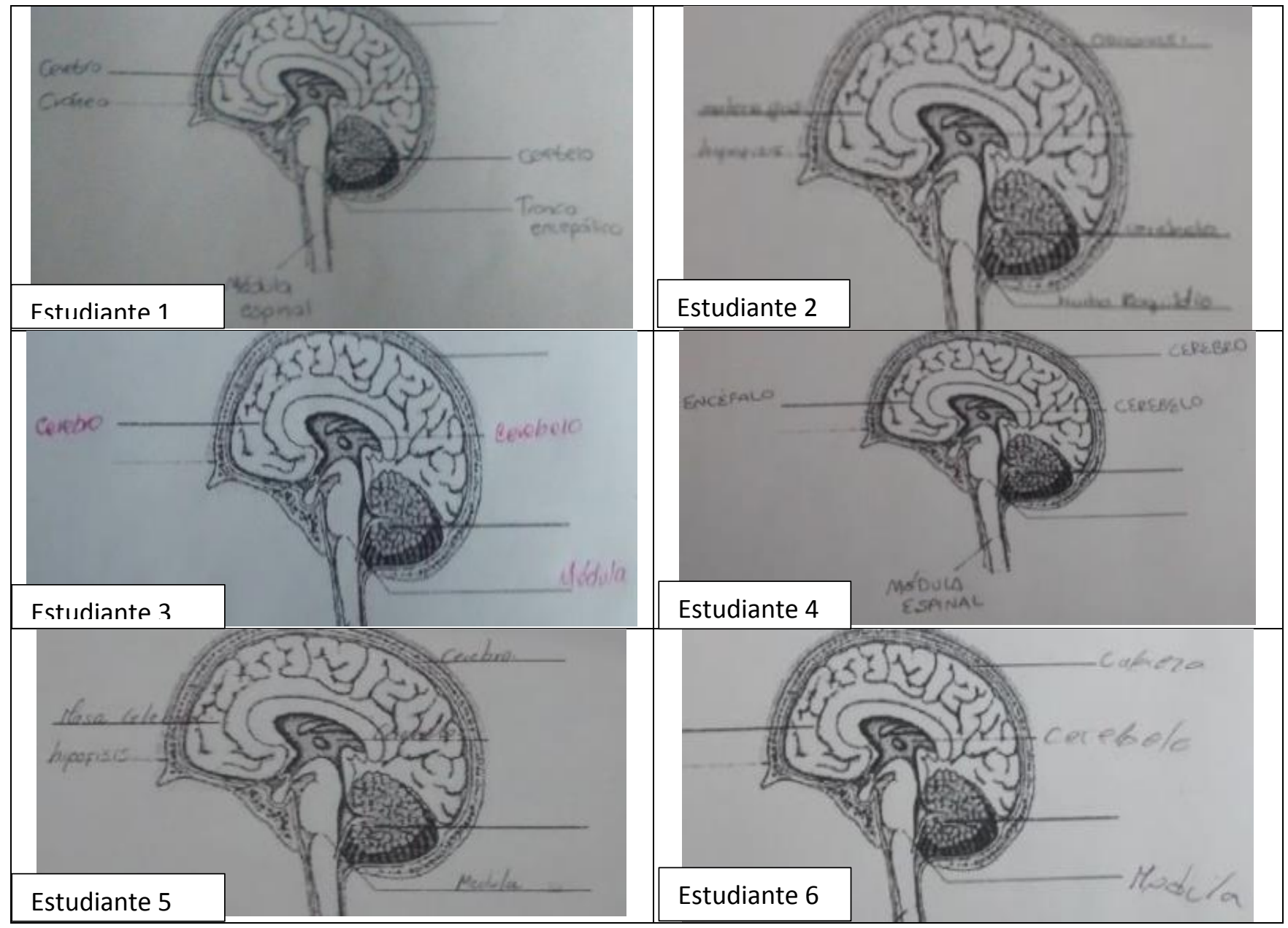

${ }^{*}$ Corresponde a la pregunta: "Si una de las funciones del oído es encargarse del equilibrio ¿cómo es posible que un sordo o un sordo ciego puedan caminar con normalidad?" ** Corresponde a la pregunta: "Colocar las partes del sistema nervioso central señaladas"

Sesión 2: En esta sesión se logró una teorización acerca del sistema nervioso comparado, sin embargo, al iniciar la sesión los estudiantes sostenían que el sistema nervioso de otros organismos era similar al del humano (Tabla 4). Se abordaron las diferencias y semejanzas entre diversos grupos taxonómicos, apoyados en una guía que sirvió como forma de estudio. Antes de entrar a explicar Vertebrados se hizo una retro alimentación, a continuación se expone la explicación del estudiante 1 en cuanto a las diferencias y semejanzas de los Poríferos, Cnidarios, Anélidos, Artrópodos:

"Los poriferos tienen un sistema nervioso primitivo, recibe estímulos por cada célula; en los anélidos hay un cordón nervioso y ganglios cerebroides, tienen tacto, gusto y les afecta la luz; y los Artrópodos tienen un cordón nervioso que se divide por cada pata y parte del animal" 
Memorias del VIII Encuentro Nacional de Experiencias en Enseñanza de la Biología y la Educación Ambiental. III Congreso Nacional de Investigación en Enseñanza de la Biología.

Esto mostró que si hubo una claridad en cuanto a las estructuras de los diferentes grupos abordados. En la parte final de la sesión se realizó otra pregunta para lograr establecer las diferencias y similitudes entre los grupos de vertebrados: " $¿ E s$ el sistema nervioso de peces, anfibios, reptiles (Aves) y mamíferos similar o presentan alguna diferencia? (Ver tabla 5), varios estudiantes intervinieron afirmando que si habían, sin embargo notaron las estructuras similares. Lo anterior, nos lleva a deducir que los estudiantes tienden a relacionar estructura y función de manera muy estrecha.

Tabla 4. Respuestas de los estudiantes que se tuvieron durante la sesión de clase de teorización.

\begin{tabular}{|c|c|c|}
\hline \multicolumn{3}{|r|}{ Sesión 2 - inicio } \\
\hline Pregunta & Estudiante & Respuesta \\
\hline \multirow{4}{*}{$\begin{array}{l}\text { “Cómo es el } \\
\text { sistema nervioso } \\
\text { en Poríferos, } \\
\text { Cnidarios, } \\
\text { Anélidos, } \\
\text { Artrópodos y } \\
\text { Vertebrados?" }\end{array}$} & Estudiante 1 & $\begin{array}{l}\text { "Debe haber diferencias ya que son animales } \\
\text { diferentes" }\end{array}$ \\
\hline & \begin{tabular}{|l|} 
Estudiante 2 \\
Estudiante 3
\end{tabular} & "Debe ser igual al de nosotros" \\
\hline & Estudiante 4 & "Es como el de nosotros pero de diferente tamaño" \\
\hline & \begin{tabular}{|l|} 
Estudiante 5 \\
Estudiante 6
\end{tabular} & "No se profe" \\
\hline
\end{tabular}

Tabla 5. Respuestas de los estudiantes que se tuvieron durante la sesión de clase de teorización.

\begin{tabular}{|c|c|c|}
\hline \multicolumn{3}{|r|}{ Sesión 2 - final } \\
\hline Pregunta & Estudiante & Respuesta \\
\hline \multirow{6}{*}{$\begin{array}{l}\text { “Es el sistema } \\
\text { nervioso de } \\
\text { peces, anfibios, } \\
\text { reptiles (Aves) y } \\
\text { mamíferos } \\
\text { similar a } \\
\text { presentan alguna } \\
\text { diferencia? }\end{array}$} & Estud & $\begin{array}{l}\text { "Profe, yo creo } \\
\text { van volviendo } m\end{array}$ \\
\hline & Es & $\begin{array}{l}\text { "Yo creo que responden dife } \\
\text { lugares diferentes, aunque ter }\end{array}$ \\
\hline & & "Son iguales, $\mathrm{p}$ \\
\hline & Estur & "No se \\
\hline & Estu & $\begin{array}{l}\text { "El sistema nervioso de los peces debe ser muy } \\
\text { simple, pero yo creo que hace lo mismo que en los } \\
\text { otros animales" }\end{array}$ \\
\hline & Estudiante 6 & "El de los mamíferos debe tener más órganos, profe" \\
\hline
\end{tabular}

Sesión 3: En esta sesión se acercó a los estudiantes al trabajo en un laboratorio de biología, reconocieron los hábitos que se deben tener para trabajar en un laboratorio: el uso de bata, el manejo del kit de disección, los estudiantes se ubicaron en los mesones del laboratorio, disponiendo esto para realizar la disección.Se tuvo en cuenta lo explicado en la sesión 1 con respecto a las funciones de las partes del 
Memorias del VIII Encuentro Nacional de Experiencias en Enseñanza de la Biología y la Educación Ambiental. III Congreso Nacional de Investigación en Enseñanza de la Biología.

sistema nervioso central y en la 2 sesión con respecto a las aves, debido a que la disección era de encéfalo de ave. Establecieron relaciones entre los órganos y sus funciones, de igual manera al finalizar la práctica hallaron diferencias entre el encéfalo de un ave y el del humano. A partir este comprendieron la anatomía del encéfalo de aves, y luego de la identificación de cada parte del encéfalo lograron definir la función de cada uno de estos (Ver Tabla 6), algo que anteriormente no se había logrado, esto demuestra la importancia que tienen los trabajos prácticos en la construcción de conceptos. Inicialmente los estudiantes empezaron a realizar la disección, atendiendo a las explicaciones de los docentes, posteriormente cada uno de los estudiantes participo en la actividad y a medida que la realizaban se les preguntó acerca de la función de cada una de las estructuras que conformaban el encéfalo. Finalmente, La disección por parte de los estudiantes permitió identificar y reconocer estructuras tales como: cerebro, bulbo olfatorio, cerebelo, tronco encefálico y medula espinal (Ver Figura 1), los estudiantes concluyeron que las estructuras del ave no presentaban mayor complejidad.

Tabla 6. Definiciones de los estudiantes ante las estructuras reconocidas en la disección del encéfalo de ave.

\begin{tabular}{|c|c|}
\hline \multicolumn{2}{|l|}{ SESIÓN 3} \\
\hline EST & ESTRUCTURAS Y FUNCIONES \\
\hline Estudiante 1 & $\begin{array}{l}\text { "El cerebro ayuda a los movimientos, el cerebelo da el equilibrio con } \\
\text { el oído interno, el tronco encefálico ayuda a que el corazón funcione, } \\
\text { la médula reparte nervios al cuerpo" }\end{array}$ \\
\hline Estudiante 2 & $\begin{array}{l}\text { "El cerebro en las aves es menos desarrollado y permite moverse, el } \\
\text { cerebelo permite el equilibrio, la médula es larga y recibe impulsos } \\
\text { rápidos, el tronco encefálico da movimientos involuntarios" }\end{array}$ \\
\hline ante 3 & $\begin{array}{l}\text { "El ave tiene un sistema nervioso más simple, las partes son más } \\
\text { pequeñitas, La médula reparte los nervios por el cuerpo, El bulbo } \\
\text { olfatorio esta frente al cerebro, el oído interno ayuda al equilibrio con } \\
\text { el cerebelo" }\end{array}$ \\
\hline 4 & $\begin{array}{l}\text { "Tenemos el sistema nervioso más desarrollado que las aves, las } \\
\text { aves tienen grandes los ojos y se unen al nervio óptico, el cerebelo }\end{array}$ \\
\hline Estudiante 5 & $\begin{array}{l}\text { permite que haya equilibrio, el tronco encefálico controla el corazón, } \\
\text { la médula se une con el tronco y recibe los impulsos" }\end{array}$ \\
\hline Estl & $\begin{array}{l}\text { "El cerebro de la gallina no tiene un tamaño tan grande y ayuda a los } \\
\text { movimientos, la médula está en la columna y reparte nervios, el } \\
\text { cerebelo deja estar en equilibrio con el oído interno" }\end{array}$ \\
\hline
\end{tabular}


Memorias del VIII Encuentro Nacional de Experiencias en Enseñanza de la Biología y la Educación Ambiental. III Congreso Nacional de Investigación en Enseñanza de la Biología.

Figura 1. Disección del encéfalo de ave realizada por los estudiantes.

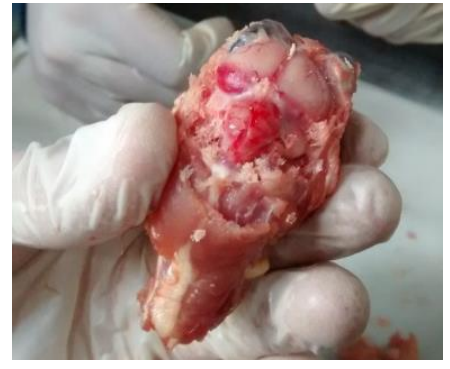

\section{CONCLUSIONES}

La contribución de los ejercicios prácticos a la construcción de conceptos resulta de vital importancia ya que acerca a los estudiantes a procedimientos habituales del trabajo de laboratorio, además que permite corroborar la teoría que es vista en el aula de clase. El ejercicio de la disección por parte de los estudiantes requirió de una teorización previa que dio lugar a una familiarización de estructuras las cuales se iban a identificar en el laboratorio, es decir que para lograr un correcto ejercicio práctico se debe tener bases que justifique dicha actividad, sino se tienen estas bases el ejercicio se mostraría de forma vacía ya que los estudiantes no establecerían relaciones de ningún tipo. Se debe tener en cuenta las ideas previas de los estudiantes debido a que estas orientaran las diversas dinámicas que van a influir en el desarrollo de la totalidad de las clases. A partir de estas se desarrolló el tema de sistema nervioso comparado, con este se logró que los estudiantes identificaran las similitudes y diferencias entre diversos organismos, transformando la idea que todos los organismos presentan sistema nervioso similar al de un humano. Por último, en la sesión práctica se logró evidenciar que hubo un cambio conceptual.

\section{REFERENCIAS}

Caamaño, A. (2004) Experiencias, experimentos ilustrativos, ejercicios prácticos e investigaciones: ¿una clasificación útil de los trabajos prácticos?. Alambique, 39(8).

Furió, C. y Gil, D., (1989) La didáctica de las ciencias en la formación inicial del profesorado: una orientación y un programa teóricamente fundamentados. Enseñanza de las Ciencias, 7(3), pp. 257-265.

García, S; Mondelo, A y Martínez, M. (1995) El trabajo práctico. Una intervención para la formación de profesores. Enseñanza de las Ciencias. 13, pp. 203-209.

Gil, D ; Carrascosa, J; Furió, C. y Martínez, J. (1991) La Enseñanza de las ciencias en la Educación Secundaria. Universitat de Barcelona.

Giordan, A (1987) Los conceptos de biología adquiridos en el proceso de aprendizaje. Enseñanza de las Ciencias. 5(2), pp, 105-110.

González, E. (1992) ¿Qué hay que renovar en los trabajos prácticos?. Enseñanza de las Ciencias. 10, pp. 206-211. 
Bio -grafía. Escritos sobre la Biología y su Enseñanza. ISSN 2027-1034

Edición Extraordinaria. p.p.1360-1370

Memorias del VIII Encuentro Nacional de Experiencias en Enseñanza de la Biología y la Educación Ambiental. III Congreso Nacional de Investigación en Enseñanza de la Biología.

Lledó, A. y Cañal, P. (1993). El diseño y desarrollo de materiales curriculares en un modelo investigativo. Investigación en la Escuela, 21, pp. 9-19.

Olson, J. (1990) The Student Laboratory and the Science Curriculum. Edited by Elizabeth Hegarty-Hazel. Editorial Routledge. Londres.

Tamir, P. (1989) Training teachers to teach effectively in the laboratory. Science teacher Education, 73(1), pp. 59-69. 